\title{
Etude comparative de deux produits de neem (huile et poudre) sur les stades préimaginaux du moustique Culex quinquefasciatus (Diptera : Culicidae)
}

\section{Fawrou SEYE*, Raymond Demba NDIONE et Mady NDIAYE}

Unité d'entomologie, rickettsiologie, bactériologie et virologie (UERBV), Laboratoire de biologie de la reproduction, Département de biologie animale, Faculté des sciences et techniques, Université Cheikh Anta Diop, BP 5005 Dakar-Fann (Sénégal).

(Reçu le 14 Décembre 2005, accepté le 17 Mai 2006)

*Correspondance, courriel : fawrou@yahoo.fr

\section{Résumé}

Des produits de neem (Azadirachta indica) (huile de neem formulée $1 \%$ et poudre de neem $0,3 \%$ ) fabriqués par une industrie sénégalaise (SENCHIM), ont été appliqués sur des larves et nymphes du moustique (Culex quinquefasciatus). Les doses ont été de 0,02 à $0,038 \%(v / v)$ et de 0,2 à $2 \%(p / v)$ respectivement pour l'huile de neem formulée et la poudre de neem.

Les résultats dans les conditions du laboratoire ont montré que la poudre de neem était plus toxique au stade larvaire (mortalités entre 86,1 et $100 \%$ ) qu'au stade nymphal (entre 14,5 et 95,9\% d'adultes envolés). Cependant, l'huile de neem formulée a été efficace aussi bien au stade larvaire (mortalité entre 52,1 et $80 \%$ ) qu'au stade nymphal (entre 0 et $14 \%$ d'adultes envolés). L'étude histopathologique a montré que les produits agissent au niveau du système digestif des larves après ingestion alors qu'au niveau des nymphes seul l'effet contact est observé. L'huile de neem formulée $1 \%$ apparaît plus efficace que la poudre de neem 0,3 \% pour le contrôle des moustiques en milieu aquatique.

Mots-clés : neem, Azadirachta indica, stade préimaginal, Culex quinquefasciatus.

\section{Abstract}

Comparative study of two neem products (oil and powder) on preimaginal stages of Culex quinquefasciatus mosquito (Diptera: Culicidae)

Neem (Azadirachta indica) products (neem oil formulated $1 \%$ and neem powder $0.3 \%$ ), manufactured by a Senegalese industry (SENCHIM), were applied to mosquito (Culex quinquefasciatus) larvae and nymphs. The doses products were from 0.02 to $0.038 \%$ 
(neem oil) and 0.2 to $2 \%$ (powder). In laboratory conditions, results showed that neem powder was more toxic at larval stage (mortalities between 86.1 and $100 \%$ ) than nymphal stage (between 14.5 and $95.9 \%$ adults flow away). Neem oil formulated was effective as well as larval stage (mortality between 52.1 and $80 \%$ ) than nymphal stage (between 0 and $14 \%$ adults flown away). The histopathological study reveals that neem products act upon larval digestive system after ingestion, whereas for nymphs only contact effects are showed. Neem oil formulated $1 \%$ appears more effective than neem powder $0.3 \%$ for mosquitoes control in aquatic environment.

Keywords : Neem, Azadirachta indica, preimaginal stage, Culex quinquefasciatus.

\section{Introduction}

Malgré leur efficacité dans la lutte contre les insectes et plus particulièrement contre les moustiques, l'utilisation des insecticides chimiques de synthèse présente des inconvénients par l'apparition des effets indésirables [1-3]. L'utilisation des produits naturels devient alors une perspective de recherche et fait l'objet de plusieurs investigations. Dans le cadre de la recherche d'insecticides naturels, le neem figure parmi les plantes les plus utilisées. Le neem (Azadirachta indica) est un arbre d'origine Asiatique que l'on trouve souvent dans les régions arides et semi-arides. Au niveau de cette plante, la quantité d'huile et des principaux triterpenoïdes varient périodiquement au cours de l'année [4]. Les amandes de neem sont riches en triterpènes et en huile.

Différents produits sont formulés à partir de l'huile de neem (Neemix, Margosan-0, etc.) et utilisés contre les insectes. Son caractère insecticide a été étudié sur les larves de certains moustiques tels que Culex sp. [5], Anopheles sp [6], Aedes aegypti [7]. La principale matière active est l'azadirachtine. La teneur en matière active est plus élevée dans les graines que dans le reste de la plante [6]. Après pressage des amandes de neem, la totalité de la matière active reste dans les tourteaux alors que l'huile n'en possède qu'une faible teneur. La poudre est généralement utilisée par les jardiniers pour la protection des cultures. Le neem a des caractères antiappétant, répulsif et inhibiteur de la croissance des insectes. Des extraits aqueux de neem ont montré des effets néfastes sur la fertilité, la fécondité et le développement post-embryonnaire de certains diptères [8]. Les travaux de [9] ont montré que l'huile des amandes de neem est plus efficace contre l'oviposition et contre l'émergence des adultes de l'insecte Callosobruchus maculatus que la poudre de neem.

En milieu urbain et périurbain, la prolifération des moustiques en particulier celle du Culex quinquefasciatus est observée en période hivernale et post-hivernale. L'utilisation des produits de neem (huile et poudre) contre les larves de moustiques pourrait réduire 
cette prolifération. Cependant, pour les stades préimaginaux des moustiques, la phase nymphale est plus courte que la phase larvaire. De ce fait, l'efficacité et l'action de ces deux produits peuvent différer selon le stade préimaginal. Il est donc nécessaire de faire une étude comparative de ces deux produits sur les stades préimaginaux des moustiques.

La présente étude consiste à :

- préciser le stade le plus sensible pour chaque produit,

- montrer à quel niveau agissent les deux produits pour chaque stade,

- proposer le produit le plus efficace pour lutter contre la prolifération des moustiques.

\section{Matériel et méthodes}

\section{2-1. Matériel}

\section{2-1-a. Matériel animal}

Des œufs du moustique Culex quinquefasciastus sont récoltés quotidiennement au niveau des bacs artificiels du département de Biologie Animale. Les œufs sont mis à éclosion dans des bacs contenant de l'eau du robinet. Après éclosion, les larves de première génération sont nourries avec de la poudre de pain en prenant le soin de changer régulièrement l'eau d'élevage. Ainsi, nous avons obtenu successivement des larves de stades $L 1, L 2, L 3, L 4$ et des nymphes.

\section{2-1-b. Matériel végétal}

Le matériel végétal nous a été fourni par la SENCHIM, une industrie chimique Sénégalaise.

\section{2-2. Méthodes}

\section{2-2-a. Traitement avec l'huile de neem formulée}

Pour chaque traitement, nous avons utilisé 11 bocaux de $10 \mathrm{~cm}$ de côté et $7 \mathrm{~cm}$ de hauteur contenant chacun $500 \mathrm{~mL}$ d'eau. Pour les larves, nous avons mis dans chaque bocal 100 larves dont 25 individus pour chaque stade larvaire et de la poudre de pain comme nourriture. Pour les nymphes, le même nombre d'individus est utilisé.

Le volume en huile de neem formulée varie de $0,02 \%$ à $0,038 \%$ avec des pas de $0,002 \%$.

Nous avons fait des constats de mortalité toutes les $24 \mathrm{~h}$ en faisant chaque jour le cumul des mortalités. 


\section{2-2-b. Traitement avec la poudre de neem}

La quantité de poudre varie de $0,2 \%$ à $2 \%$ avec des pas de $0,2 \%$. Ces doses sont choisies suite à des tests antérieurs que nous avons effectués au laboratoire.

Le même nombre d'individus est utilisé aussi bien pour les larves que pour les nymphes. Nous avons fait des constats de mortalité toutes les $24 \mathrm{~h}$ en faisant chaque jour le cumul des mortalités.

Pour les traitements, la température moyenne pour les traitements était de $26^{\circ} \mathrm{C} \pm 0,55$ et le taux d'humidité moyenne était de $88 \% \pm 0,14$.

\section{2-2-c. Analyse statistique}

Nous avons effectué deux séries de traitements et les valeurs données représentent la moyenne des deux séries. Les mortalités corrigées sont calculées en appliquant la formule d'Abbott :

$$
\% m=(N L M-N L M T) \times 100 /(N T L-N L M T)
$$

$\% \mathrm{~m}=$ pourcentage de mortalité

$\mathrm{NLM}=$ nombre de larves mortes

NLMT $=$ nombre de larves mortes dans le témoin

$\mathrm{NTL}=$ nombre total de larves

Pour les nymphes, nous avons considéré en même temps la mortalité des nymphes et des adultes issus des nymphes traitées. Pour connaître le pourcentage d'adultes envolés, nous avons fait la différence entre le nombre de nymphes traitées et le nombre d'individus morts.

Le pourcentage d'adultes envolés est obtenu en faisant cette différence :

$$
\% \text { A.E. }=1-\%(N . M .+ \text { A.M. })
$$

$\%$ A.E. $=$ Pourcentage d'adultes envolés

N.M. $=$ Nymphes Mortes

A.M. $=$ Adultes Morts

Le logiciel Excel est utilisé pour le calcul des moyennes de mortalité pour chaque stade. Pour chaque produit, nous avons utilisé le test Khi-deux $\left(X^{2}\right)$ pour voir si l'efficacité des produits entre les larves et les nymphes est significativement différente ou non.

\section{2-2-d. Etude histologique}

Après traitement, les larves et nymphes moribondes sont fixées au carnoy2 pendant $72 \mathrm{~h}$. Les larves sont ensuite soumises successivement à une déshydratation, imprégnation et inclusion. Après confection et la taille des blocs, les coupes de $7 \mu \mathrm{m}$ d'épaisseur faites au 
microtome sont colorées par la méthode du trichome de Masson. Les observations sont faites au microscope photonique Motic relié à un micro-ordinateur où les photos sont enregistrées sous format JPEG.

\section{Résultats}

3-1. Effets insecticides des produits de neem sur les larves de $C$. quinquefasciatus

\section{3-1-a. Cas de l'huile de neem formulée}

Tableau 1 : Pourcentages de mortalités des larves de Culex quinquefasciatus traitées avec l'huile de neem formulée $1 \%$

\begin{tabular}{|c|c|c|c|c|c|c|c|c|c|c|c|}
\hline \multirow{2}{*}{ Jours } & \multicolumn{7}{|c|}{ Pourcentages en huile de neem formulée (1 \%) } & \multirow{2}{*}{ Témoin } \\
\cline { 2 - 11 } & 0,02 & 0,022 & 0,024 & 0,026 & 0,028 & 0,03 & 0,032 & 0,034 & 0036 & 0,038 & \\
\hline J1 & 24,5 & 27,5 & 24,5 & 22 & 25 & 16 & 22 & 24,5 & 25 & 41,5 & 2 \\
\hline J2 & 32 & 43 & 41 & 47 & 40 & 52 & 43,5 & 43 & 55,5 & 58,5 & 3 \\
\hline J3 & 37,5 & 51 & 52,5 & 60 & 51 & 58,5 & 56 & 54 & 67 & 62 & 8 \\
\hline J4 & 43 & 51,5 & 54 & 66,5 & 56,5 & 61,5 & 57,5 & 58 & 72 & 69 & 8 \\
\hline J5 & 48,5 & 54 & 58,5 & 74,5 & 60 & 67,5 & 59,5 & 60 & 74,5 & 70,5 & 9 \\
\hline J6 & 51 & 56 & 62,5 & 77,5 & 64,5 & 68,5 & 62 & 61,5 & 77 & 74 & 10 \\
\hline J7 & 52,5 & 62,5 & 67,5 & 82,5 & 68,5 & 71,5 & 63,5 & 63,5 & 80 & 77,5 & 11 \\
\hline
\end{tabular}

Le Tableau 1 montre une mortalité croissante des larves de Culex quinquefasciatus au cours du temps. Ce tableau montre des mortalités allant de 52,5 à $80 \%$ sept jours après traitement par rapport au témoin (11\% de mortalité). Cependant, nous n'avons pas obtenu $100 \%$ de mortalité. Une semaine après test, la moyenne des mortalités est de $68,95 \%$. 


\section{3-1-b. Cas de la poudre de neem}

Tableau 2 : pourcentages de mortalités des larves de Culex quinquefasciatus traitées avec la poudre de neem $0,3 \%$

\begin{tabular}{|c|c|c|c|c|c|c|c|c|c|c|c|}
\hline \multirow{2}{*}{$\begin{array}{c}\text { jour } \\
\mathrm{s}\end{array}$} & \multicolumn{8}{|c|}{ Pourcentages en poudre de neem $(0,3 \%)$} & \multirow{2}{*}{ Témoin } \\
\cline { 2 - 11 } & 0,2 & 0,4 & 0,6 & 0,8 & 1 & 1,2 & 1,4 & 1,6 & 1,8 & 2 & \\
\hline $\mathrm{Jl}$ & 1,5 & 4,5 & 4,5 & 4,5 & 8 & 13,5 & 13 & 11 & 10,5 & 16 & 0 \\
\hline $\mathrm{J} 2$ & 5,5 & 8 & 16 & 22,5 & 27,5 & 41,5 & 53 & 65,5 & 74,5 & 74 & 0 \\
\hline $\mathrm{J} 3$ & 12 & 30 & 39,5 & 51 & 50 & 69 & 69,5 & 77 & 86 & 79 & 0 \\
\hline $\mathrm{J} 4$ & 37,6 & 42,7 & 74,8 & 64,7 & 65,7 & 76,2 & 78,3 & 98,4 & 98,4 & 96,4 & 0,5 \\
\hline $\mathrm{J} 5$ & 62,9 & 68,5 & 83,7 & 82,7 & 94,8 & 95,4 & 93,3 & 100 & 100 & 100 & 1,5 \\
\hline $\mathrm{J} 6$ & 68,8 & 80 & 83,8 & 85,7 & 95,8 & 96,4 & 96,9 & 100 & 100 & 100 & 2 \\
\hline $\mathrm{J} 7$ & 86,1 & 87,1 & 95,8 & 90,2 & 96,8 & 96,8 & 98,9 & 100 & 100 & 100 & 2,5 \\
\hline
\end{tabular}

Le Tableau 2 montre une mortalité croissante des larves de Culex quinquefasciatus au cours du temps mais aussi avec les doses $(0,2$ à $2 \%)$. Les doses létales $50(1,4 \%)$ et 100 $(2,6 \%)$ sont obtenues respectivement en 2 et 5 jours. Une semaine après, la moyenne des mortalités est de $95,17 \%$.

3-2. Effets insecticides des produits de neem sur les nymphes de $C$. quinquefasciatus

\section{3-2-a. Cas de l'huile de neem formulée}

Tableau 3 : pourcentages d'adultes envolés en fonction de la quantité d'huile de après traitement des nymphes de Culex quinquefasciatus

\begin{tabular}{|c|c|c|c|c|c|c|c|c|c|c|c|}
\hline \multirow{2}{*}{ Jours } & \multicolumn{7}{|c|}{ Pourcentages en huile de neem formulée (1 \%) } & Témoin \\
\cline { 2 - 11 } & 0,02 & 0,022 & 0,024 & 0,026 & 0,028 & 0,03 & 0,032 & 0,034 & 0,036 & 0,038 & \\
\hline J2 & 0,6 & 2,2 & 7,9 & 14 & 12,9 & 8,1 & 0 & 0 & 0 & 0 & 84,5 \\
\hline
\end{tabular}

Le Tableau 3 montre une nette diminution des pourcentages de moustiques adultes envolés (entre 0 et $14 \%$ ) en fonction des doses en huile de neem par rapport au témoin $(84,5 \%)$. La moyenne des mortalités $\left(m_{1}\right)$ est de $95,43 \%$ par rapport au témoin 
$(13,5 \%)$. A partir de la dose $0,032 \%$, aucun adulte ne s'est envolé. La majeure partie des individus traités est morte 2 jours après.

\section{3-2-b. Cas de la poudre de neem}

Tableau 4 : pourcentages d'adultes envolés en fonction de la quantité poudre de neem après traitement des nymphes de Culex quinquefasciatus

\begin{tabular}{|c|c|c|c|c|c|c|c|c|c|c|c|}
\hline \multirow[t]{2}{*}{ Jours } & \multicolumn{10}{|c|}{ Pourcentages en poudre de neem $(0,3 \%)$} & \multirow[t]{2}{*}{ Témoin } \\
\hline & 0,2 & 0,4 & 0,6 & 0,8 & 1 & 1,2 & 1,4 & 1,6 & 1,8 & 2 & \\
\hline J2 & 95,9 & 87,7 & 86,6 & 80,5 & 81,5 & 53,7 & 40,3 & 20,7 & 25,8 & 14,5 & 97 \\
\hline
\end{tabular}

Le Tableav 4 montre une diminution des pourcentages de moustiques adultes envolés (entre 14,5 et 95,5\%) en fonction de la teneur en poudre par rapport au témoin (97\%). Cependant cette diminution est plus faible par rapport à l'huile de neem formulée. La moyenne des mortalités $\left(m_{2}\right)$ est de $41,28 \%$ et donc la majeure partie des individus est passée en adulte et s'est envolée.

\section{3-3. Comparaison de l'efficacité de chaque produit sur les deux stades préimaginaux}

\section{3-3-a. Avec l'huile de neem formulée}

L'application du test Khi-deux montre que l'efficacité de l'huile de neem formulée sur les larves et les nymphes n'est pas significativement différente (ddl $=9 ; \alpha=5 \%$; $X^{2}$ tabulé $=3,325 ; X^{2}$ calculé $=1,133$ ).

\section{3-3-b. Avec la poudre de neem}

L'application du test Khi-deux montre que l'efficacité de la poudre de neem sur les larves et les nymphes est significativement différente $\left(\mathrm{ddl}=9 ; \alpha=5 \% ; \mathrm{X}^{2}\right.$ tabulé $=3,325$; $X^{2}$ calculé $\left.=8,59\right)$. 
3-4. Etude histologique des larves et nymphes traitées aux produits de nem

3-4-a. Sur les larves

* Larves non traitées
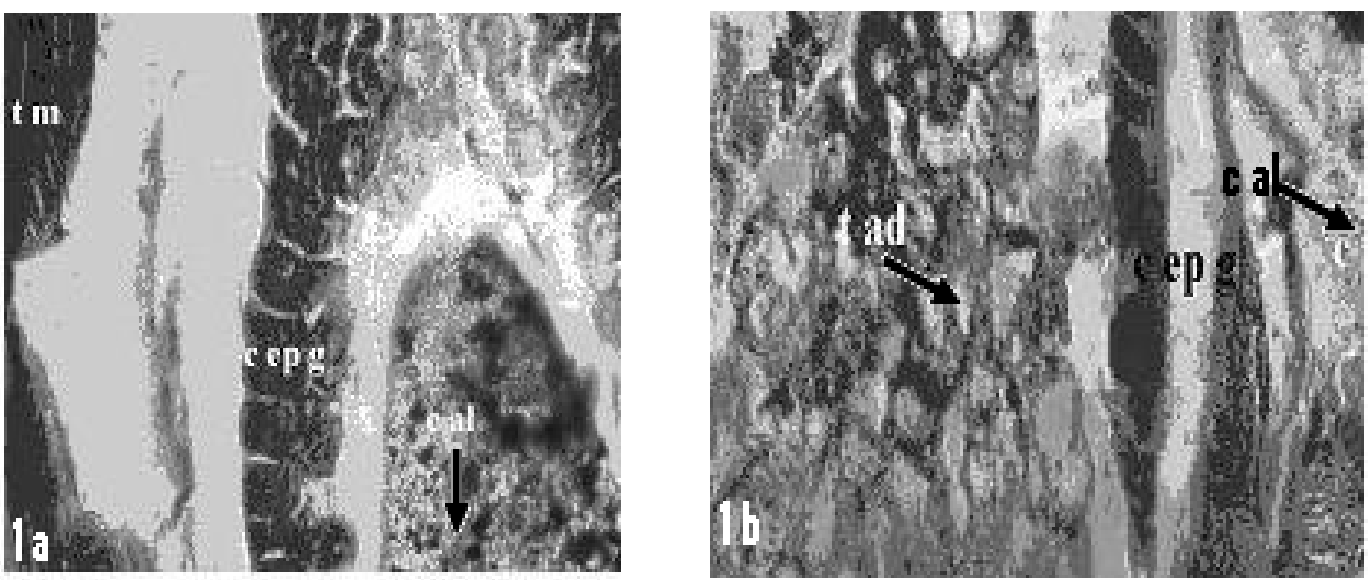

Figures $1 \mathbf{a} \& \mathbf{l b}:$ Coupes histologiques des larves de Culex quinquefasciatus non traitées (témoin) $(G=400)$

La coupe longitudinale passant au niveau du thorax des larves de Culex quinquefasciatus non traitées montre la présence de tissus intacts (tissu adipeux $(t$ ad), tissu musculaire $(t$ $\mathrm{m}$ ). La colonne alimentaire (c al) est présente et est entourée par les cellules épithéliales gastriques (c ep g).

* Larves traitées avec l'huile de neem formulée

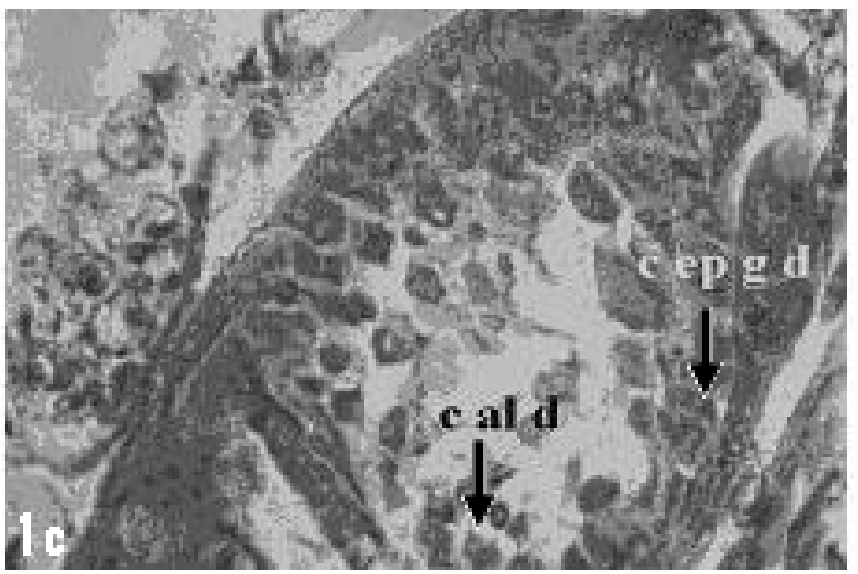

Figure 1c: Coupe histologique d'une larve de Culex quinquefasciatus traitée à l'huile de neem formulée $(G=x 400)$

Fawrou Seye et al. 
La coupe longitudinale au niveau antérieure des larves de Culex quinquefasciatus traitées à l'huile de neem formulée (lc) montre, des cellules épithéliales gastriques détruites ( $c$ ep $g d$ ) envahissant la lumière du caecum, une colonne alimentaire désorganisé (c al d) et un tissu adipeux absent par rapport au témoin (Figures la et lb).

\section{* Larves traitées avec la poudre de neem}

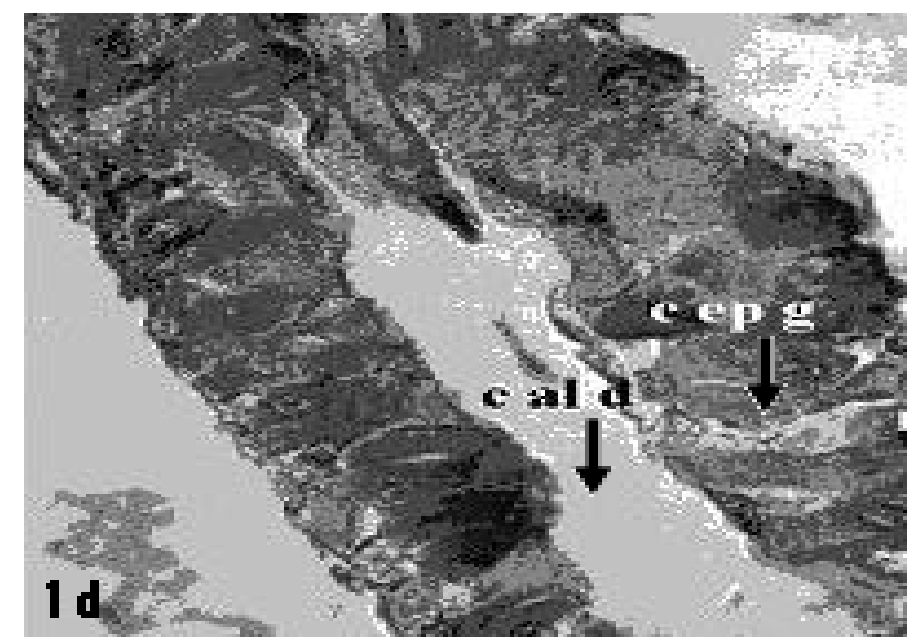

Figure 1d : Coupe histologique d'une larve de Culex quinquefasciatus traitée à la poudre de neem $(G=\times 400)$

La coupe longitudinale au niveau antérieure des larves de Culex quinquefasciatus traitées à la poudre de neem montre une destruction des cellules épithéliales gastriques ( $c$ ep g) et une désorganisation de la colonne alimentaire ( $c$ al d). Cependant, la désorganisation des cellules gastriques est moins importante par rapport à celle des larves traitées à l'huile.

\section{3-4-b. Sur les nymphes}

\section{* Nymphes non traitées (témoin)}

Sur les Figures $\mathbf{2 a}$ et $\mathbf{2} \boldsymbol{b}$, nous pouvons noter la présence des cellules glandulaires ( $\mathrm{gl}$ ), de la chaîne nerveuse (ch $\mathrm{n})$, du tissu musculaire $(\mathrm{t} \mathrm{m})$ et des réserves alimentaires ( $r$ al) en bon état. 

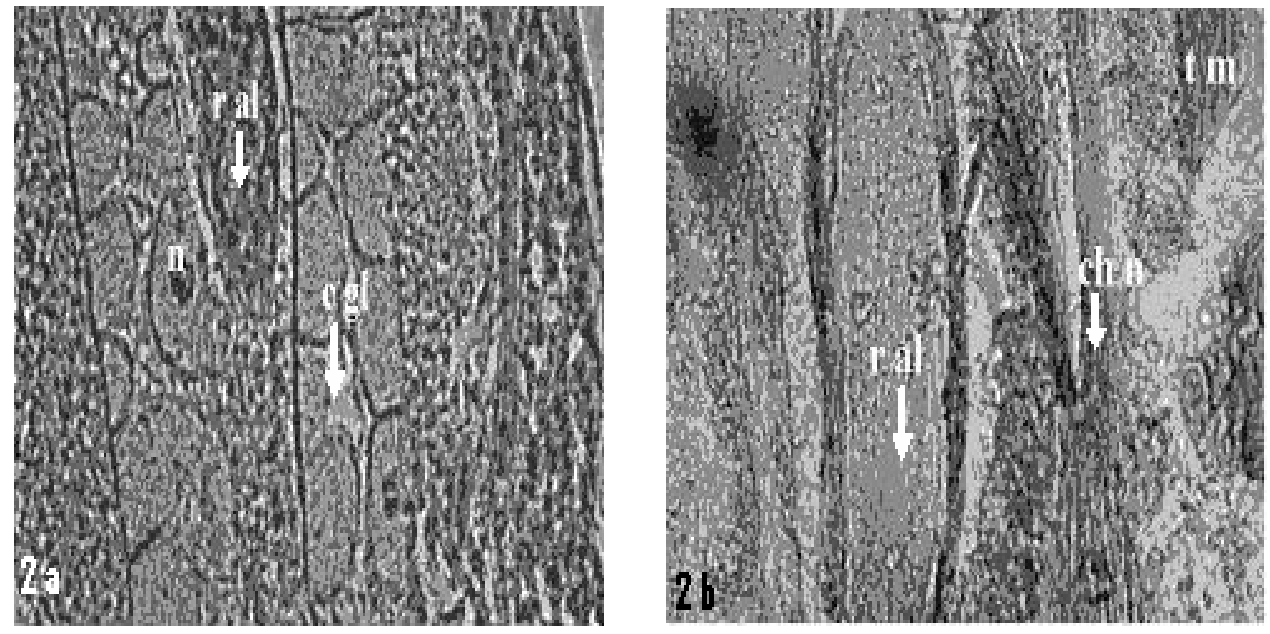

Figures 2a et 2b : Coupes histologiques des nymphes de Culex quinquefasciatus non traitées (témoin) $(G=x$ 400)

* Nymphes traitées avec l'huile de neem formulée

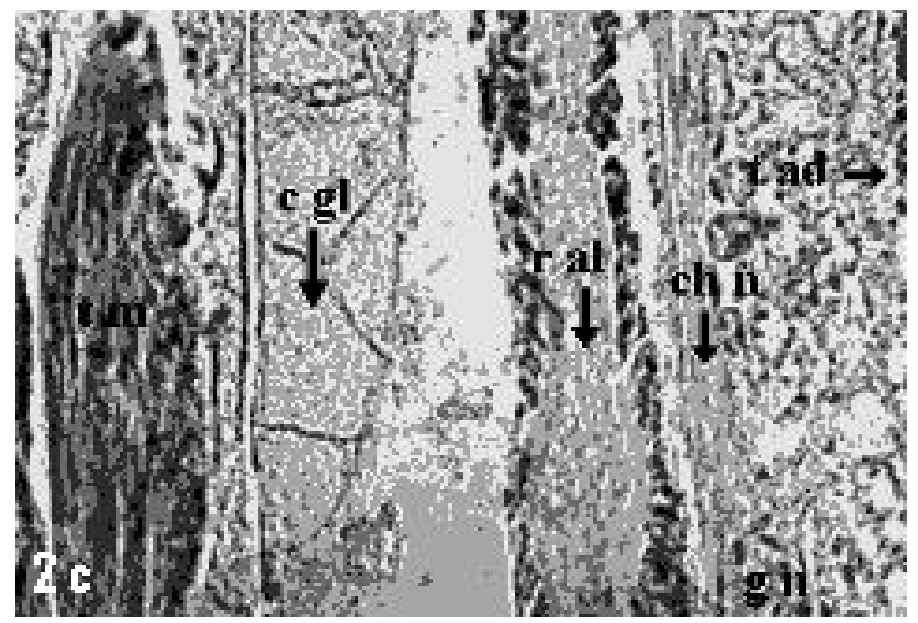

Figure 2c : Coupe histologique d'une nymphe de Culex quinquefasciatus traitée à l'huile de neem formulée $(G=x 400)$.

Sur cette Figure $2 \boldsymbol{c}$, nous pouvons noter que les organes observés à savoir les cellules glandulaires (c gl), la chaîne nerveuse (ch $\mathrm{n})$, le tissu musculaire $(\mathrm{t} \mathrm{m})$, le tissu adipeux ( $t$ ad) et les réserves alimentaires $(r$ al), Cependant, nous pouvons noter un début de dégradation des structures par rapport au témoin (Figures $2 a$ et $2 b$ ). 
* Nymphes traitées avec la poudre de neem

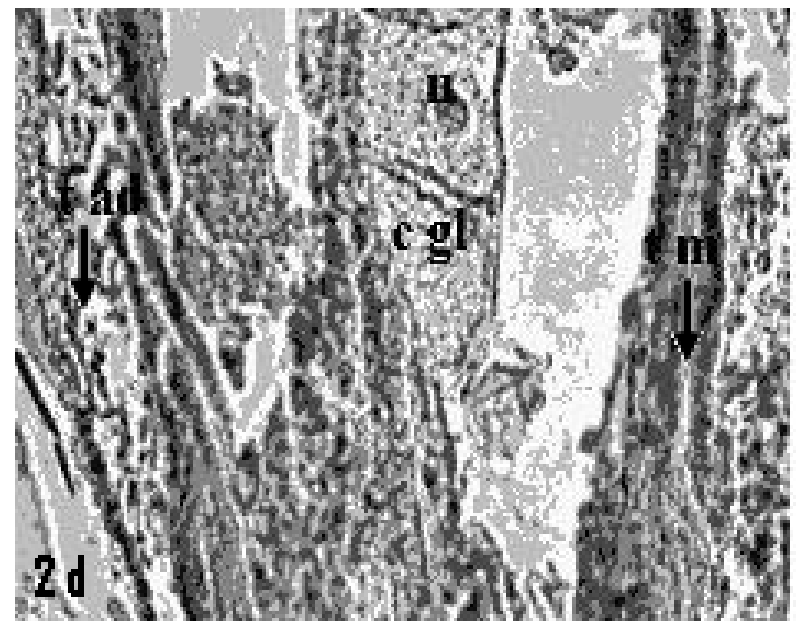

Figure 2d : Coupe histologique d'une nymphe de Culex quinquefasciatus traitée à la poudre de neem ( $G=400)$

Sur la Figure $2 \boldsymbol{d}$, nous pouvons noter la présence du tissu adipeux ( $t$ ad), des cellules glandulaires ( $\mathrm{c} \mathrm{gl}$ ) avec leur noyau ( $\mathrm{n}$ ) et du tissu musculaire $(\mathrm{t} \mathrm{m})$. Cependant, nous pouvons noter un début de dégradation des structures par rapport au témoin (Figures 2a et 2b)

\section{Discussion}

De nombreux travaux ont montré l'efficacité de l'huile de neem sur les insectes $[5-7,10]$. Cependant, il faut noter qu'en plus de l'action de la matière active, l'huile de neem pure tue les larves de moustique par asphyxie en les empêchant de respirer à la surface de l'eau.

La formulation de l'huile de neem pure en y ajoutant des sulfactants lui permet d'être miscible avec l'eau. Les travaux de [11] ont montré une mortalité de $51,6 \%$ des larves de Culex quinquefasciatus en un jour avec $5 \%$ d'huile de neem émulsionnable. Avec l'huile de neem formulée $1 \%$, nous avons obtenu pour une dose de $0.03 \%$ (soit $3 \mathrm{mg} / \mathrm{L}$ en azadirachtine) $50 \%$ de mortalité en 2 jours (Tableav 1).

Selon [12], la moitié de la durée de vie de l'azadirachtine est de $282 \mathrm{~h}$ à $25^{\circ} \mathrm{C}$ et celle-ci décroît si la température augmente. Nous avons effectué nos travaux dans des conditions de température comprises entre 24 et $28,5^{\circ} \mathrm{c}$ durant 7 jours.

Les résultats montrent l'effet toxique des produits de neem sur le développement des

Fawrou Seye et al. 
larves et nymphes de moustique. Cependant, l'huile et la poudre ne se comportent pas de la même manière. L'huile de neem formulée est aussi efficace contre les larves que contre les nymphes. Ce qui n'est pas le cas pour la poudre de neem. En effet, la matière active (azadirachtine) qui se trouve dans la poudre (solide) est libérée lentement dans l'eau alors que la formulation de l'huile facilite la libération et la diffusion de la matière active. Les fortes mortalités des larves observées (100\%) avec l'application de la poudre de neem (Tableau 2) sont dues au fait que les doses choisies sont plus fortes que celles de l'huile de neem formulées. Au bout de 2 jours, la majeure partie de la matière active est passée du solide (poudre) vers le liquide (eau). La durée des quatre stades larvaires (LI, L2, L3 et L4) étant plus longue, la matière active peut donc agir avant que les larves ne puissent passer en nymphes.

Les travaux de [13] ont montré que l'application de la poudre de neem à $500 \mathrm{~kg} / \mathrm{ha}$ ou 50 $\mathrm{g} / \mathrm{m}^{2}$ a entrâné une mortalité des larves de Culex quinquefasciatus à $90 \%$ dans des champs de riz. Considérant nos bocaux ayant une surface de $0,01 \mathrm{~m}^{2}(10 \times 10 \mathrm{~cm})$, nous pouvons faire une correspondance de cette dose qui serait à $0,5 \mathrm{~g}$ pour $0,01 \mathrm{~m}^{2}$. Dans nos travaux, cette dose correspond à celle ayant donné une mortalité située entre 87 et 95 $\%$ en 7 jours. Cette similitude montre bien l'efficacité de la poudre de neem sur les larves de moustiques aussi bien au laboratoire qu'en milieu ambiant.

Le stade nymphal des moustiques dure entre 2 et 3 jours, ce qui fait que les nymphes traitées avec la poudre de neem auront le temps de passer en adulte et de s'envoler avant que le produit n'agisse. Avec l'huile, la mortalité des individus (nymphes et adultes émergés) est plus importante $\left(m_{1}=95,43 \%\right)$ qu'avec la poudre de neem $\left(m_{2}=42,28 \%\right)$. En effet, lors de nos travaux, nous avons constaté que certaines nymphes traitées à l'huile de neem formulée sont mortes avant la nymphose, d'autres au moment de la nymphose (effet contact). Nous avons pu constater que certains adultes issus des nymphes traitées à l'huile de neem n'ont pas pu s'envoler. Ce qui serait du à la nature huileuse qui empêche les moustiques de s'envoler après émergence.

Avec la poudre de neem, la mortalité des nymphes est observée pratiquement au moment de la nymphose (effet contact du produit). Sur le plan physiologique, les nymphes ne se nourrissent pas mais utilisent des réserves nutritives du stade larvaire. Chez les larves, les produits appliqués agissent après ingestion (destruction des cellules gastriques) et par effet contact, alors que chez les nymphes, seul l'effet contact se manifeste. En effet, puisque les nymphes ne se nourrissent pas, les produits ne peuvent donc pas être ingérés. Contrairement aux larves qui en s'alimentant ingèrent en même temps le produit appliqué. Le mode d'action de ces produits sur les cellules gastriques est identique à celui décrit par [14]. L'application de la poudre de neem ne peut empêcher aux nymphes d'évoluer en adulte que si la dose est forte. L'utilisation de l'huile de neem formulée en milieu aquatique est donc plus sûre pour empêcher la 
prolifération des moustiques. Néanmoins, la poudre reste toujours efficace contre les larves du moustique Culex quinquefasciatus. L'huile de neem formulée et la poudre de neem agissent de la même manière au niveau des larves (destruction des cellules gastriques) mais différemment au niveau des nymphes (effets contacts au moment et après la nymphose).

\section{Conclusion}

Les résultats de notre étude nous ont permis de dire que l'huile de neem formulée est aussi efficace sur les larves que sur les nymphes. La poudre par contre n'est efficace que sur les larves.

L'huile de neem formulée et la poudre de neem agissent par voie intestinale et par contact sur les larves, alors qu'elles n'agissent que par contact sur les nymphes.

A l'issue de cette étude, nous pouvons dire que l'huile de neem formulée est plus efficace que la poudre de neem sur les stades préimaginaux des moustiques.

\section{Références}

[1] - M.D. FerRANDO., E. SANCHOA., E ANDREU-MOLINER, "Chronic toxicity of fenitrothion to an algae (Nannochloris oculata), a rotifer (Brachionus calyciflorus), and the cladoceran (Daphnia magna)". Ecotoxicology Environment Safety, 35 (2) (1996) 112-120

[2] - F NUWAHA, "The challenge of chloroquine-resistant malaria in sub-Saharan Afric", Health policy and planning, 16 (1) (2001) 1-12

[3] - Y.L KONAN., A.A KOFFI., J.M.C DOANNIO., et F. DARRIET, "Résistance de Culex quinquefasciatus (SAY, 1823) à la Deltaméthrine et l'utilisation de la moustiquaire imprégnée en milieu urbain de Bouaké", Bull. soc. pathol. Exot., 96 (2) (2003) 128-129

[4] - S JOHNSON, E. D. MORGAN et N. P. COLIN, "Development of the major triterpenoids and oil in the fruit and seed of neem (Azadirachta indica)", Annals of Botany, 78 (1996) 383-388

[5] - M. SCOTT, et N.K. KAUSHIK, "The toxicity of a neem insecticide to populations of culicidae and other aquatic invertebrates as assessed in situ microcosms", archives of environment Contamination and Toxicology, 39 (3) (2000) 329-336

[6] - B.L. ALIERO, "Larvaecidal effects of aqueous extracts of Azadirachta indica (neem) on the larvae of Anopheles mosquito", African Journal of biotechnology, 2 (9) (2003) 325-327 
[7] - B. CAROLINA, WANDSCHEER, E. JONNY, DUQUE, M.A.N. DA SILVA, F YOSHIYAS., J. L. WOHLKE, J ADELMANN. and J. D FONTANA, "Larvaecidal action of ethanolic extract from fruit endocarps of Melia azedarach and Azadirachta indica against the dengue mosquito Aedes aegypti", Toxicon, 44 (8) (2004) 829-835

[8] - S. SHIVENDRA., "Effects of aqueous extract of neem seed Kernel and azadirachtin on the fecundity, fertility and post-embryonic development of the melonfly, Bactrocera cucurbitae and the oriental fruit fly, Bactrocera dorsalis (Diptera: Tephritidae)", Journal of Applicata. Entomology, 127 (2003) p. 540

[9] - N.E.S.A LALE, "Evaluation of neem (Azadirachta indica A. Juss) seed oil obtained by different methods and neem powder for the management of Callosobruchus maculatus (F.) (Coleoptera: Bruchidae) in stored cowpea", Journal of Stored Products Research, 35 (2) (1999) 135-143

[10] - B.S. ATTRI et G.R PRASAD, "Neem oil extractive and effective mosquito larvicide", Indian Journal Entomology, 42 (1980) 371-374

[11] - C.P. BATRA, P.K MITTAL, T. ADAK., V.P SHARMA, "Efficacy of neem oil-water emulsion against mosquito immatures", Indian Journal Malarial, 35 (1) (1998) 15-21

[12] - S.Y. SZETO et M.T WAN, "Hydrolysis of Azadirachtin in buffered and natural waters", Journal Agriculture Food Chemistry, 44 (1996) 1160-1163

[13] - D.R RAO, R REUBEN, M.S VENUGOPAL, B.A NAGASAMPAGI, H. SCHMUTTERER "Evaluation of neem, Azadirachta indica, with and without water management, for the control of culicine mosquito larvae in rice-fields", Med. Vet. Entomology, 6 (4) (1992) 318-24

[14] - HK Koua, SH Han, M.A. d'Almeida. "Histopathology of Anopheles gambiae s.l. Giles, 1902 (Diptera, Culicidae) subjected to the larvicidal activity of the aqueous extract of Persea americana Miller, 1768 (Lauraceae)". Bull Soc Pathol. Exot; 91 (3) (1998) 252-256 